\title{
A Review On Variable MEMS Mirrors For Photo-Lithographic Masks
}

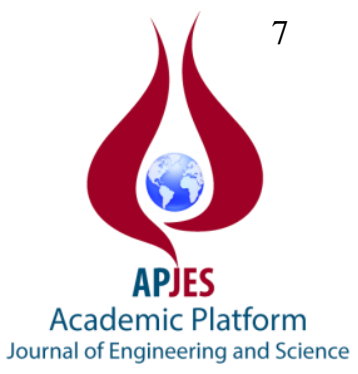

\begin{abstract}
Mehmet Akif Erişmiş
Necmettin Erbakan Üniversitesi

Electrical and Electronics Engineering Department

Address: Necmettin Erbakan University, Electrical and Electronics Engineering Department maerismis@konya.edu.tr

E-mail address:

Abstract

This paper presents a review on using MEMS based mirror arrays to achieve maskless lithography in order to eliminate mask costs in the micro fabrication processes. With the advanced technology nodes, it becomes more and more costly to produce the lithography masks. Especially with the extreme ultraviolet lithography technique, the necessity to use maskless lithography becomes more obvious. Several universities and companies fabricated tilting mirror style or piston style mirror arrays to propose a solution to maskless lithography processes.
\end{abstract}

Keywords: Micromirror arrays, Maskless Lithography, MEMS

Özet

Bu makale MEMS tabanlı mikro-ayna dizilerinin mikro-üretim adımlarında maske maliyetini azaltmak için maskesiz litografi elde etmek amaçlı kullanılması hakkında bir derleme sunmaktadır. Gelişmiş teknoloji nodlarıtla birlikte litografi maskelerini üretmek daha da pahalı olmaktadır. Özellikle EUV (ekstrim ultraviolet litografi) tekniğinde, maskesiz litografi kullanmak ihtiyacı daha da aşikar olmuştur. Maskesiz litografi adımlarına bir çözüm olması amacıyla, çeşitli üniversite ve şirketler dönen veya piston şeklinde mikro-ayna dizileri üretmiş̧ir.

Anahtar Kelimeler: Mikro-ayna dizileri, Maskesiz litografi, MEMS

\section{Introduction}

The fabrication of IC's and MEMS sensors consists of several deposition and etching steps. The substrate is first coated with a photo sensitive material, photoresist, and then this photoresist is patterned with lithography. The patterned photoresist serves as the masking layer for successive process steps. Hence, lithography is one of the key process steps in the microfabrication area. Photo-lithography, or optical lithography, is the widely used lithography technique, because of its simplicity, low cost, and high through-put [1].
Figure 1 shows two simplified configurations of optical lithography process. In Figure 1a, the mask patterns are directly imaged to the substrate without any demagnification steps. This method is convenient if the minimum dimensions to be patterned are in $\mu \mathrm{m}$ levels. However, if the desired patterns are sub- $\mu \mathrm{m}$ level, then the configuration

Doi:10.5505/apjes.2013.54264 
shown in Figure $1 \mathrm{~b}$ is used with a demagnification factor of usually $4: 1[2]$.

The clock frequency of the processors and the maximum memory cells to be placed in a fixed area are directly related with how small a transistor can be fabricated. Hence, the IC fabrication industry continuously seeks to miniaturize the transistor sizes, and the silicon microfabrication technology has enabled this miniaturization for years. Indeed, this miniaturization is guessed even in 1965, by G.E.Moore. Although his first guess is that the number of transistors in the state-of-the-art IC will double in each year, the average doubling time turned out to be 18 months [3]. (a)

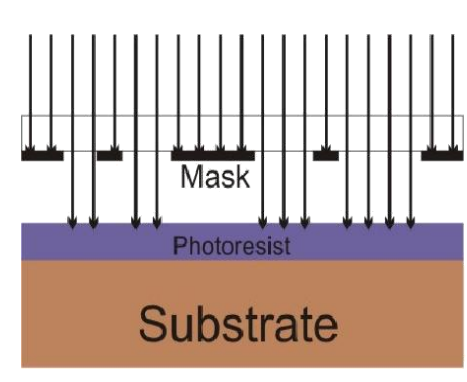

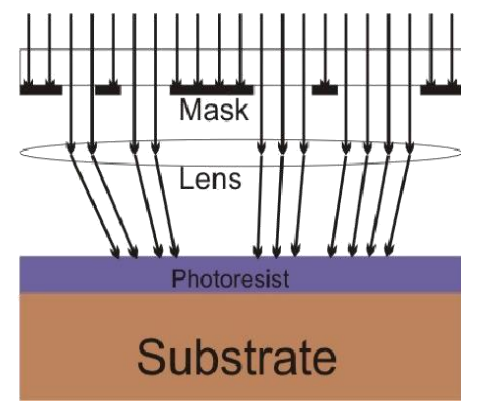

(b)
Figure 1: Two simplified configurations of optical lithography process, without any demagnification (a) and with demagnification (b).

The miniaturization brings several problems to be solved. Firstly, the mask generation becomes a problem because of very small pattern sizes. Second problem is the resolution after the exposure. Generating patterns on the mask does not guarantee that the pattern will be transferred successfully to the photoresist. The resolution issue can be better understood if the related equations are investigated:

$$
\operatorname{Re} s=K_{1} \frac{\lambda}{N A}
$$

$$
D O F=K_{2} \frac{\lambda}{(N A)^{2}}
$$

Two important criterion of lithography, Resolution (Res) and Depth of Focus (DOF) are related to incident light beam wavelength $\lambda$, and the numerical aperture of the lens system, NA. The $\mathrm{K}$ factors are process, environment, and resist dependent parameters that can change from time to time. To achieve a better resolution, $\lambda$ should be decreased and NA should be increased. However, this leads a smaller DOF. Still the scientists try to decrease $\lambda$ and arrange NA accordingly to achieve an enhanced resolution and a reasonable DOF $[2,4]$.

\section{Needs for Maskless Optical Lithography}

There are several motivations to eliminate the masks in the optical lithography step. First of all, as the minimum features in the IC's get smaller and smaller, the fabrication costs for masks become a real problem. A set of 25 to 30 masks for 130 -nm-thick lines costs US $\$ 500000$. For 90 $\mathrm{nm}$-lines the cost is expected to be more than US \$1 million. This much money may not be problematic for big companies but severely damages small ASIC design companies. Hence, especially ASIC companies are looking forward to eliminating the masks and their costs from their process flow [5].

Fabrication time of the mask sets also gets problematic as the minimum sizes get smaller. Customization an IC chip requires several fabrication cycles due to design optimizations, and each fabrication cycle will require a different mask set. Hence, customizing an IC chip requires several waiting periods for the fabrication of the mask sets. As the minimum sizes in these masks sets become smaller and smaller, a defect-free mask set for that high resolution will be more problematic, and hence time consuming.

Several new motivations for maskless optical lithography are arising with the new generation optical lithography ideas. Figure 2 shows the Extreme Ultraviolet Lithography system which is thought to be the new generation optical lithography technique. EUV uses extremely small wavelength incident light beam in the levels of $11 \mathrm{~nm}-14 \mathrm{~nm}$ to achieve an enhanced resolution. However, in this region, all known the materials, including air molecules are strongly absorbing. Hence, not only the refracting optics used for demagnification, but also the masks should also be replaced by reflecting optics. Moreover, as the air also absorbs EUV light, the exposure should be done under vacuum. In addition, it is not an easy task to find a good reflector for this low wavelength light beam [2,4]. These will be new problems of the new generation optical lithography tool, and each problem creates another motivation for maskless optical lithography.

It is not an easy task to find a good reflector in the EUV region. Scientists propose so called, distributed Bragg 
reflectors, which is composed of stacked Si/Mo thin layers. Figure 3 shows the Mo/Si stacked layer that is proposed for the EUV lithography and its reflectivity [2]. It can be seen that, even this proposed reflector is not a good reflector because it reflects only $75 \%$ of the incident light beam. Moreover, the fabrication of this reflector is not an easy and low cost process. Still a defect free $\mathrm{Mo} / \mathrm{Si}$ mask fabrication is investigated, however, the cost of this mask is expected to be US \$120000, and a mask set of 25-30 masks will be more than US \$3 million [7, 8].

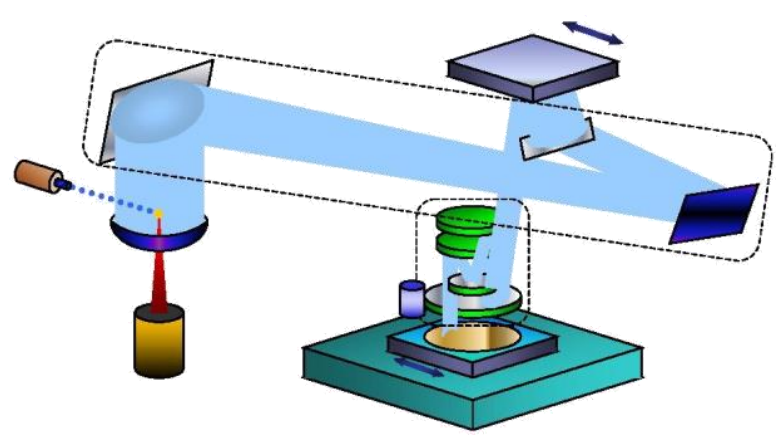

Figure 2: EUV lithography system. The system uses all reflecting optics because in EUV region all materials are strongly absorbing [6].
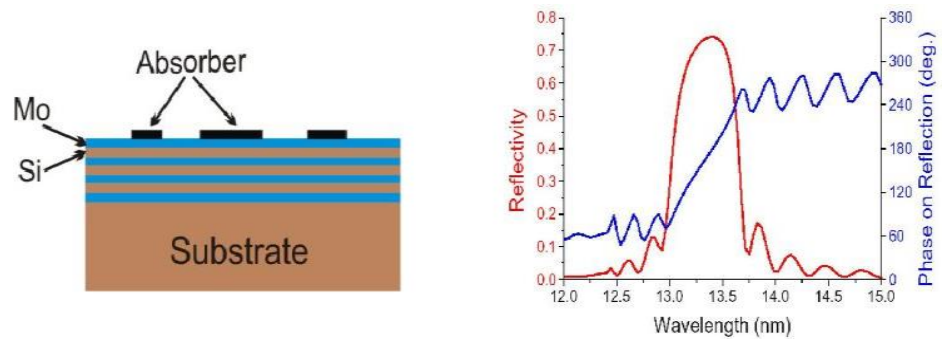

(a)

(b)

Figure 3: Mo/Si stacked layer for EUV optical lithography mask (a), and its reflectivity graph (b) [2].

Mask handling in EUV optical lithography also creates some problems for solid masks. In EUV region, all the materials are strongly absorbing; hence it is very difficult to coat a protective pellicle onto the mask. Consequently, the mask will be very fragile to environmental effects and particulation. A good solution to this problem may be to protect the mask in vacuum environment, but this time putting the mask into vacuum environment exposure chamber for exposure will be a time consuming and impractical task $[8,9]$.

Solution of these entire mask problems is thought to be eliminating all the masks in the process flow and replacing them with computer controlled Spatial Light Modulators (SLM). Using MEMS mirrors as SLM is an easy and effective way and this method is started to be commercialized by a cooperation of Swedish and Dutch companies. The idea is constructing lithography tools with embedded MEMS SLM mirrors, and programming the MEMS SLM mirrors accordingly for each individual exposure. Cost, mask fabrication time, and mask handling problems will be solved automatically with this approach. Even the defect problem can be minimized with a good computer controlled exposure, letting multiple exposures from different individual mirror elements. The major disadvantage of this approach is the throughput; however, the final products will be available earlier in the market due to eliminating mask fabrication times [5].

\section{MEMS Mirrors as Spatial Light Modulators}

MEMS micromirrors as SLM modulate the amplitude or phase of the incident light beam. One of the important usages of MEMS SLM micromirrors is in display systems. In Digital Micromirror Device, DMD, that is an early example of SLM devices designed for display systems, there are more than 400 thousand MEMS mirrors. Each mirror can be accessed individually. The mirrors are tilting type, i.e, they stay parallel to the substrate under no excitation but they can tilt up to 10 degrees in both directions when tilting voltages applied. When a light spot is wanted on the screen, the incoming light beam is reflected to the screen by tilting the corresponding mirror +10 degrees and; when a black spot is wanted on the screen the corresponding mirror is tilted by -10 degrees to reflect the incoming beam out of the screen [11]. This method is a simple, effective, and digital way of modulation of the incoming light.

Scientists thought that modulating the incoming light beam idea can also be used for optical lithography. The mask will be replaced by the MEMS SLM micromirrors. A computer program will control the general flow of the process, by controlling each individual mirror element and 
also by arranging the position of the micromirror mask relative to the wafer. As the micromirror mask should be movable to be able to expose the whole wafer, the duration of one exposure step depends on how big the mirror array is and how many mirror elements mirror array has. However, the computer program will be handling much bigger amount of data if the number of elements in the mirror array is increased. Hence a balance should be found between the process throughput and the amount of data that the computer program will handle.

Grid problem is an important issue to be discussed when we think of eliminating solid masks and replace them with MEMS SLM micromirrors. Considering that all the mirrors except one are in "OFF" state, and the remaining is in "ON" state, the minimum exposable area on the wafer (MAW) can be extracted experimentally. In fact, this area should be:

$$
M A W=\frac{M A}{D F}
$$

where MA is the mirror area and DF is the optical demagnification factor of the system. Some researchers used even this simple idea for their lithography process [12], however, the general trend is to find ways to create off-grid patterns.

There are two main design approaches for MEMS SLM micromirrors; Tilt-ing Micromirrors and Piston Micromirrors. For each of these approaches, Grayscaling method can be used for generating off-grid patterns on the wafer [13]. The following sections will explain these issues in detail.

\subsection{Tilting Micromirrors}

Figure 4 shows FhG-IMS tilting micromirror array which is being commercialized, and Figure 5 shows KTH micromirror array. In these two approaches, the mirror surface is a thin membrane staying parallel to the substrate. When proper voltages are applied between mirror membrane and the proper electrode, the membrane tilts. Hence the normal of the mirror surface changes, causing the incident beam to be reflected to another position. Figure 6 verifies the operation of the micromirror structures showing white light interferometer image of tilted FhG-IMS pixels [10, 14].

FhG-IMS micromirror array which is being commercialized has $2048 \times 512$ mirror elements which are 16 $\mu \mathrm{m} \times 16 \mu \mathrm{m}$ in dimension and fabricated from aluminum alloys. Each electrode has an analog input from an analog DRAM structure, enabling the control of tilting angle for each mirror element. This property of FhG-IMS micromirror array is important to create off-grid patterns; however, also it increases the data to be handled. This array is intended to be used for UV Lithography, not EUV Lithography, since each mirror element is too big for EUV lithography needs and also aluminum is not as a good reflector as $\mathrm{Mo} / \mathrm{Si}$ reflectors for EUV [14].

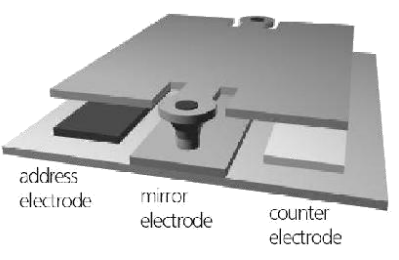

(a)

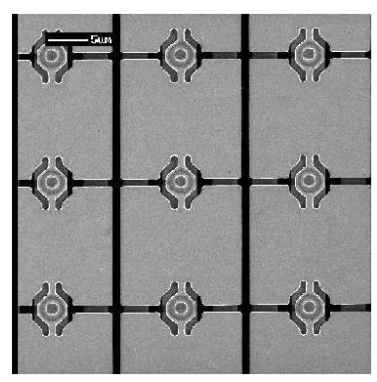

(b)
Figure 4: Schematic view of the FhG-IMS micromirror array element (a), and an SEM picture of the array (b) [14].

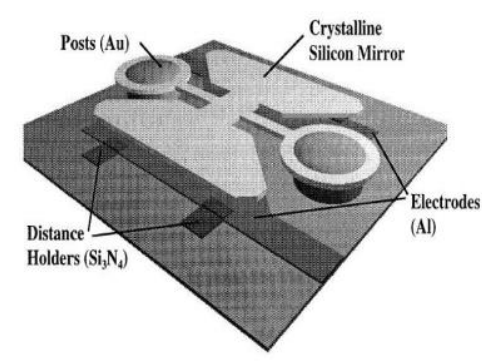

(a)

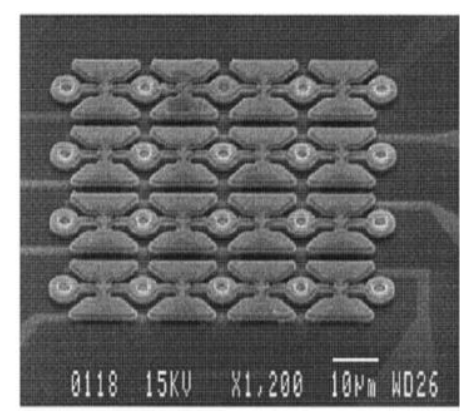

(b)
Figure 5: Schematic view of the KTH micromirror array element (a), and an SEM picture of the array (b) [10]. 


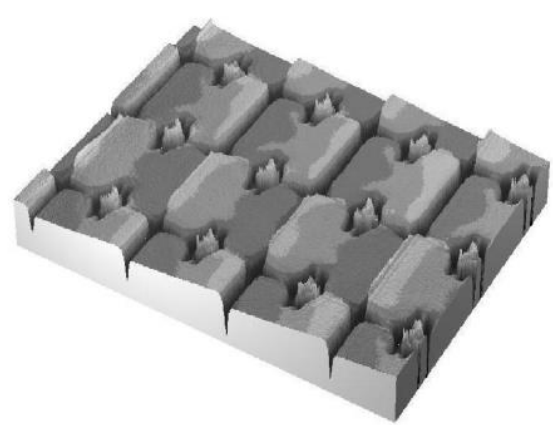

Figure 6: White light interferometer image of tilted FhG-

IMS pixels [14].

There is also research going on to achieve tilting micromirror arrays for maskless EUV lithography. Figure 7 shows the simplified view of maskless EUV lithography which uses tilting micromirrors. The major challenges for realizing micromirror arrays for this lithography are fabricating very small area mirror elements and fabricating $\mathrm{Mo} / \mathrm{Si}$ layers on top the mirror elements as EUV reflectors [8].

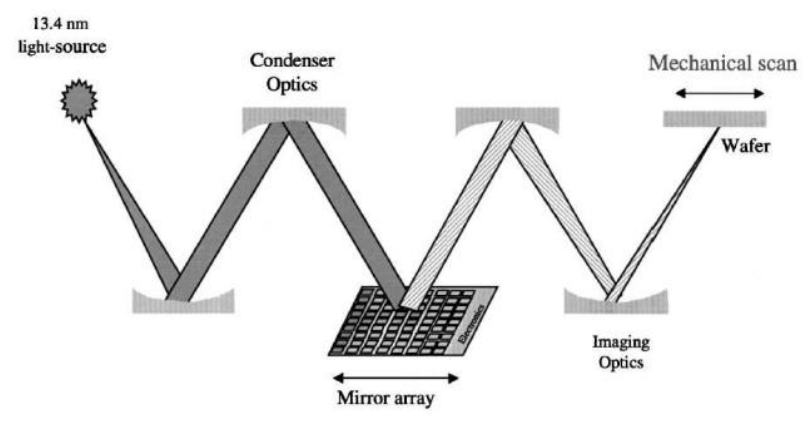

Figure 7: Simplified view of maskless EUV lithography using tilting micromirrors [8].

Figure 8 shows a tilting micromirror structure for EUV lithography pro-posed by University of Berkeley [15]. Researchers are working on the fabrication of the structure. Preliminary fabrication results are achieved, however, they are still trying to solve some fabrication problems of the nano-scale structure. The proposed area of the mirror element ranges from 0.25 to $2 \mu \mathrm{m}^{2}[15,16]$.

One of the problems arising with maskless EUV lithography is the damping of the mirror element under vacuum environment. Due to high vacuum, the quality factor of the movement of the mirror element will be quite high. This high quality factor values will result high settling times for the system, causing a decreased maximum switching frequency of the mirror. Hence, the total time for the exposure will increase due to the fact that each mirrors should expose or not expose several different areas in the wafer. Berkeley also searches for electrical damping methods to optimize the switching times of their mirror elements [17].

Figure 8: Tilting micromirror structure for EUV

lithography [15].

\subsection{Piston Micromirrors}

Piston micromirrors use the destructive interferences of the reflected incident light beam to create a light or black spot on the wafer. Figure 9 shows the formation of destructive interferences. The piston micromirror array consists of several mirror elements that stay parallel to the substrate under no excitation. When one of the mirrors is excited, it moves upwards or downwards. The magnitude of the movement is the excitation voltage dependent. When the excitation voltage is arranged so that the displacement is $\lambda / 4$ of the incident beam wavelength, there occurs $\lambda / 2$ path length difference be-tween two neighboring light beams. This path length difference creates an $180^{\circ}$ phase change and hence these light beams destructively interface [8].

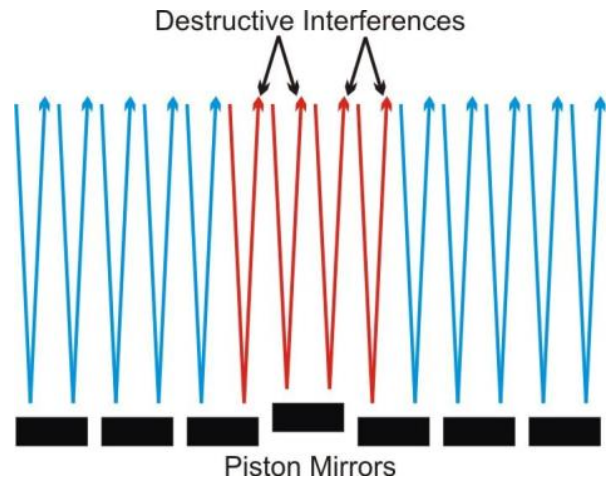

Figure 9: Destructive interference formation in piston micromirrors. 
EUV maskless lithography is the main target for piston micromirrors. Their usage for UV lithography will be problematic due to the big mirror area. However for the EUV case, the mirror areas are proposed to be very small, and hence destructive interference idea becomes more realistic in this case. Figure 10 shows the simplified view of maskless EUV lithography using piston micromirrors and a piston micromirro
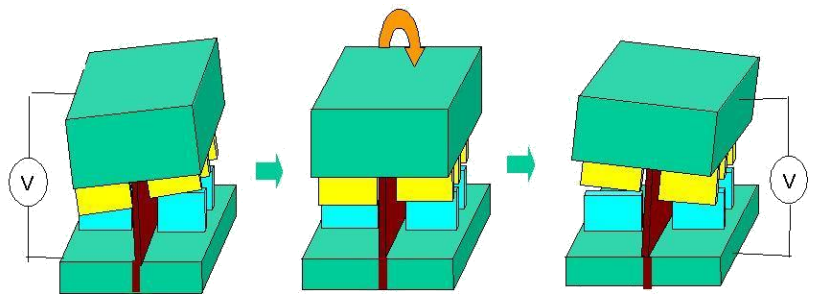

r array schematic proposed by Stanford University. The reflecting mirrors fabricated from $\mathrm{Mo} / \mathrm{Si}$ multilay-ers will be placed on an elastomer layer which is sandwiched by capacitive actuator electrodes. To actuate a micromirror array, corresponding capac-itive actuator is supplied with proper voltages, and the elastomer height changes in that region. This height change provides the deflection of the mirror element [18].

(a)

Figure 10: Simplified view of maskless EUV lithography using piston micromirrors (a) and a proposed piston micromirror array (b) [18].

University of Berkeley also proposes piston micromirror array as they propose tilting micromirror array, but they do not provide a fabrication process yet [8]. However, according to their modeling, piston micromirrors show better performance than tilting micromirrors for EUV maskless lithography process [13].

\section{Grayscaling}

Using an array of mirrors instead of a solid mask leads to achieving a very strict gridded pattern on the wafer. However, the desired pattern is generally based on a gridless design. Hence, there should be a way to create pattern edges at arbitrary places within a grid cell. Indeed grayscaling
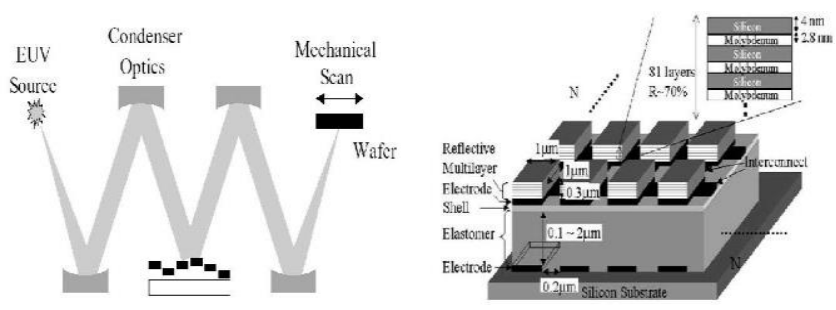

method serves this purpose very well [13].

Grayscaling method uses light beams with different intensity to expose different spots on the wafer. When the intensities of light beams of two adjacent mirrors are changed relatively, the place of the edge that is created on the wafer changes. Hence, off-grid patterns can be realized. The control over the edge place becomes more powerful depending on how many mirror elements are adjusted to create that edge. Consequently, to achieve a better control on edge place means to use a larger number of mirror elements to create it, and hence to increase the data amount to be processed. Scientists propose that two or three mirror elements for one spot will provide adequate control and will result reasonable data amounts for today's technology limitations [14].

The basic grayscaling method is to modify the exposure times of each spot on the wafer corresponding to a single mirror element. This method is easy and requires relatively small data amount but does not provide a good control. As this method does not put any extra limitation to the MEMS SLM micromirror array structure, even the basic DMD can be used as the mirror array. Indeed, researches from Carl Zeiss used this method with a DMD and presented a MEMS based lithography. They achieved $\mu \mathrm{m}$ level resolution though, due to lack of control on the places of edges [19].

A more advanced grayscaling method is to change the intensity of the reflected light beam with analog deflecting of the mirror element. Tilting mirrors use diffraction phenomena to modify the light intensity. Figure 11 shows the grayscaling for tilting mirrors and intensity vs. deflection graph for FhG-IMS tilting mirrors. As the deflection of the mirror is increased, the intensity of the light on the corresponding spot decreases due to diffraction. Hence, by an analog control on the excitation voltage, the light intensity on a given spot can be controlled [14, 20].

Two or three mirror elements can be enough to precisely modify the off-grid edge place of the pattern on the wafer. Figure 12 clearly explains this idea. Three mirrors are kept in their maximum deflection position to form black spot in some region on the wafer, while the deflection amount of the fourth one is modified. This modification gives the 
control on the edge place of the off-grid pattern on the wafer [14].

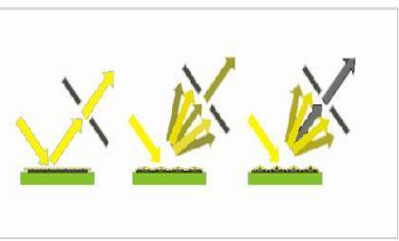

(a)

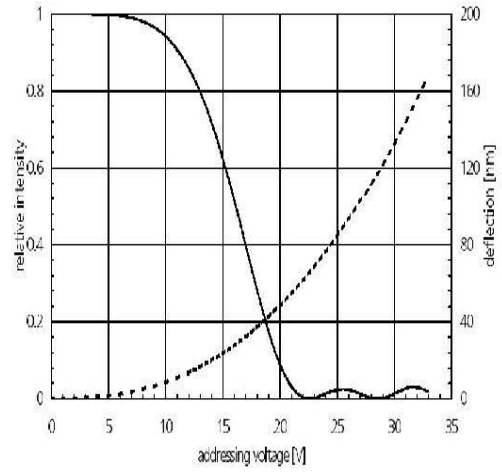

(b)
Figure 11: Grayscaling for tilting mirrors (a). The intensity vs. deflection graph for FhG-IMS tilting mirrors (b) [14, 20].

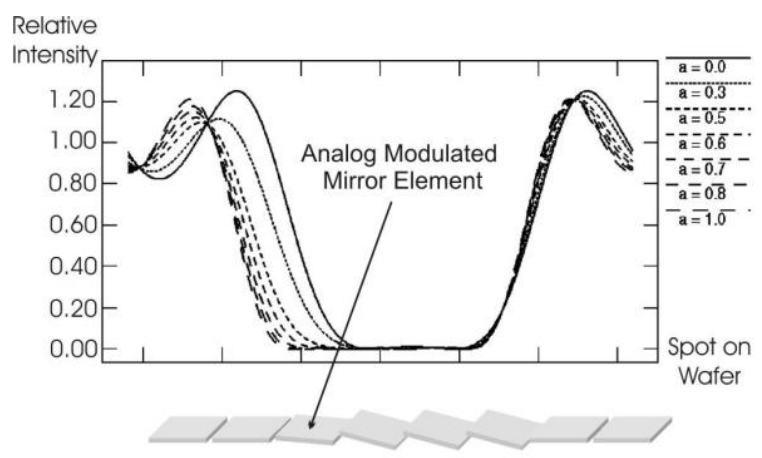

Figure 12: Off-grid edge control by analog modulation of the deflection [14].

First generation lithography processes are performed using FhG-IMS MEMS SLM tilting micromirror array with analog grayscaling. The results are very promising. Using $248 \mathrm{~nm}$ wavelength incident light beam they managed to resolve 320 $\mathrm{nm}$ minimum feature size structures. Moreover, with the help off-grid edge control, they managed control $1.6 \mathrm{~nm}$ grid offgrid edge place. Figure 13 gives some fabrication results that FhG-IMS has achieved. They manages not only lines of 200 $\mathrm{nm}$ widths, but also nano-scale non-manhattan type structures.

A further advanced analog grayscaling method uses Fourier transforms. To achieve desired patters, Fourier transform of a virtual solid mask and a micromirror array using analog grayscaling is tried to be matched. Hence, the deflection amount of each mirror element can be extracted. The feasibility of this approach is simulated, and it is shown that with using more than two mirror elements, the grid dependence of micromirror type masks can be totally eliminated [21].

Analog grayscaling is also possible for piston micromirrors. In piston mi-cromirrors, if the mirror element is deflected by $\lambda / 4$, then a black spot can be achieved on the corresponding spot in the wafer. Analog control on the deflection magnitude of the mirror element leads to analog modulation of the intensity of the reflected light beam. This method is illustrated with com-puter programs, but a fully working fabricated piston micromirror device using analog grayscaling has not been reported yet [13].

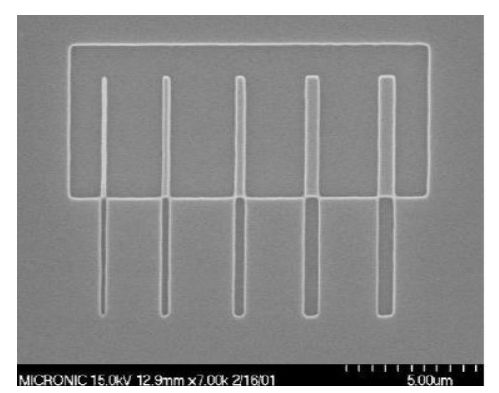

(a)

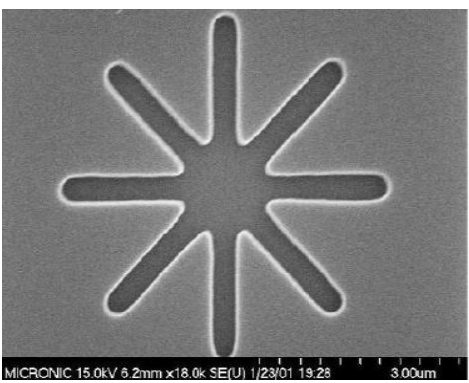

(c)

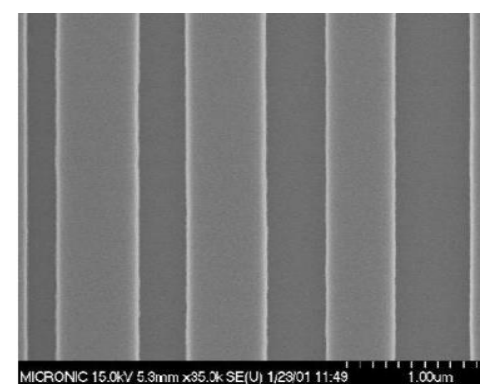

(b)

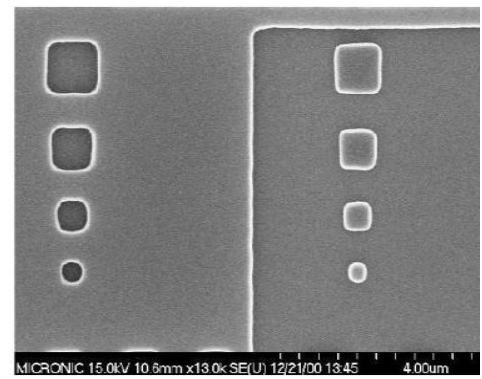

(d)
Figure 13: SEM pictures of FhG-IMS MEMS SLM tilting micromirror array optical maskless lithography. (a) and (b) shows lines of down to $200 \mathrm{~nm}$ widths. (c) shows a nanoscale non-manhattan type star shape. (d) shows isolated square shapes down to $400 \mathrm{~nm}$ widths [14]. 


\section{References}

[1] M.J.Madou, "Fundamentals of Microfabrication - The Science and Miniaturization, Second Edition", CRC Press, (2002).

[2] J.E.Bjorkholm,"EUV Lithography-The Successor to Optical Lithography?", Intel Technology Journal, (Q3), 1-8, (1998).

[3] R.R.Shaller,"Moore's Law: past, present, and future", IEEE Spectrum, 52-59, (1997).

[4] R.H.Stulen,"13-nm Extreme Ultraviolet Lithography", IEEE Journ. of Quantum Electronics 1 (3), 970-975, (1995).

[5] N.Savage,“A Revolutionary Chipmaking Technique?”, IEEE Spectrum, 18, (2003).

[6] http://www.nikon-precision.com/docs/euv.pdf

[7] C.Gwyn,"EUV Lithography Update," SPIE OE Magazine, 22-24, (2002).

[8] N.Choksi, D.S.Pickard, M.McCord, R.F.Pease, Y.Shroff, Y.Chen, W.Oldham, and D.Markle,"Maskless extreme ultraviolet lithography", Journ. of Vacuum Science and Technology, B17 (6), 3047-3051, (1999).

[9] N.Harned and S.Roux,"Progress Report: Engineers take the EUV lithography challenge", SPIE OE Magazine, 18-20, (2003).

[10] F.Niklaus, S.Haasl, and G.Stemme, "Arrays of monocrystalline silicon micromirrors fabricated using CMOS compatible transfer bonding," IEEE Journ. of MEMS, 465$469,(2003)$.

[11] J.M.Younse, "Projection Display Systems based on the digital micromirror ${ }^{\mathrm{TM}}$ device $\left(\mathrm{DMD}^{\mathrm{TM}}\right)$ )", SPIE 2641, 64-75, (1995).

[12] K-N.Lee, D.S.Shin, W-J.Chung, Y-K.Kim, and Y-S.Lee, "Protein patterning by virtual mask photolithography", IEEE Conf. on Microtechnologies in Medicine\&Biology, 136-139, (2002).

[13] Y.Shroff, Y.Chen, W.G.Oldham, "Optical Analysis of Nanomirror Based Pattern Generation for Maskless lithography", Techcon (2003).
[14] H.Lakner, P.D“urr, U. Dauderstadt, W.Doleshall, and J.Amelung, "Design and fabrication of micromirror arrays for UV-Lithography”, SPIE 4561, 255-264, (2001).

[15] Y.Chen, C.Chu, Y.Shroff, and W.G.Oldham, "Fabrication and Dynamics of Electrically-Damped Double-Comb Nanomirrors for EUV Maskless lithography," Techcon (2003).

[16] Y.Shroff, Y.Chen, and W.G.Oldham, "Fabrication of parallel-plate nanomirror arrays for extreme ultraviolet maskless lithography", Journ. of Vacuum Science and Technology, B19 (6), 2412-2415, (2001).

[17] Y.Chen, Y.Shroff, and W.Oldham, "Modeling and control of nanomirrors for EUV maskless lithography," Modeling and Simulation of Microsystems,, 602-604, (2000).

[18] J-S.Wang, I.W.Jung, and O.Solgaard,"Fabrication method for elastomer spatial light modulators for short wavelength maskless lithography," Sensors and Actuators, A 114, 52535, (2004).

[19] L.Erdmann, A.Deparney, F.Wirth, and R.Brunner,"MEMS based lithography for the fabrication of microoptical components," SPIE 5347, 79-84, (2004).

[20] www.tpd.tno.nl/smartsite210.html

[21] H.Martinsson and T.Sandstrom,"Rastering for SLM-based mask-making and maskless lithography", SPIE 5567, 557564, (2004). 